\title{
Antireflection Microstructured Surface on ZnSe for Mid-infrared Spectral Region
}

\author{
Jia-Ji Cao, Qian-Kun Li, Yan-Hao Yu, and Yong-Sen Yu* \\ State Key Laboratory on Integrated Optoelectronics, College of Electronic Science and Engineering, \\ Jilin University, Changchun 130012, Peoples Republic of China \\ E-mail: yuys@jlu.edu.cn
}

\begin{abstract}
Antireflection microstructured surface were fabricated on $\mathrm{ZnSe}$ through a rapid and scalable method which was called femtosecond laser direct writing (FsLDW). With this technology, micrometer level inverted pyramid and cone arrays were fabricated precisely. The measured transmittance were about $11.3 \%$ higher compared with the plain $\mathrm{ZnSe}$ at $9 \mu \mathrm{m}$ in the ideal situation. These results were in good accord with the simulations which were calculated by geometric and diffractive field tracing techniques.
\end{abstract}

DOI: $10.2961 /$ jlmn.2019.02.0001

Keywords: antireflection, subwavelength structure, diffractive optics, ZnSe, femtosecond laser direct writing

\section{Introduction}

Zinc selenide $(\mathrm{ZnSe})$ is an ideal and promising material for applications to the infrared band optical and electronic devices [1-4], such as window in solar battery, fairing and convex lens. This kind of material also used as laser crystal of mid-infrared solid-state laser [5], which become a hot topic in domestic and overseas research for its widespread application value and prospect in remote sensing of atmosphere, laser radar, spectroscopy, national defense and spaceflight aviation. The refractive index of $\mathrm{ZnSe}$ from 2 $\mu \mathrm{m}$ to $15 \mu \mathrm{m}$ is about 2.4 , which caused high surface reflectance $[6,7]$. In the traditional way, single- or multilayer film coating technology is used to manipulate the high and low refractive indices [8-10]. However, multiple problem may arise after the film coating [11-13]. First, the optical properties of the coating may be damaged by dust, defects, humidity or other kinds of environmental degradations. Second, the refractive index of the coating material may not match with bulk material. Most of all, the coating may cracking or shedding when the outside temperature changed dramatically, which is unsuitable for high power laser or spacecraft. Antireflection microstructured surface prepared on the material can easily overcome these problems. Compared with film coating, antireflection microstructured surface can be equivalent to a gradient film system with refractive index range from air to material [14].

After first discovered by Bernard in 1968, researchers have conducted a detailed study of antireflection microstructured surfaces [15]. This opened the way for those who did this research later. In the early nineties, with holographic approach, antireflective sub micrometer surfacerelief gratings were produced on various stand positive photoresists with an area of $190 \mathrm{~mm} \times 166 \mathrm{~mm}$ [16]. Which can produce large-area antireflection microstructures efficiently. However only quasi-sinusoidal structures can be produced with this kind of method, as well as the structure may breaks if it is way too deep. Joachim P. Spatz fabricated highly light transmissive interfaces for antireflective optics in the deep-UV range which were prepared by nanolithography and reactive ion etching [17]. With this kind of fabrication method, plan convex fused silica lenses were demonstrated with an increased transmittance of light between 185 and $300 \mathrm{~nm}$. Which were of great use in deepUV range. Whereas the experimental procedure was quite complex. Block copolymer self-assembly and plasma etching were also leveraged to fabricate enhanced broadband antireflection in silicon solar cells. Though the hydrogen bromide and chlorine used during inductively coupled plasma-reactive ion etching were not environmentally friendly. To overcome these problems, femtosecond laser direct writing (FsLDW) has the superiority in high peak power and the accuracy in three-dimensional machining which have been used in micro-nano fabrication [18-26].

In this letter, we fabricated antireflection microstructure on $\mathrm{ZnSe}$ for mid-infrared spectral range with femtosecond laser direct writing (FsLDW) technology. High accuracy inverted pyramid and cone arrays with different arrangement mode were fabricated on $\mathrm{ZnSe}$. This device is much more stable because antireflection microstructure and $\mathrm{ZnSe}$ are integrated. Which may have potential applications in infrared detection and imaging technology.

\section{Materials and Methods}

The mathematical models currently used for describing the propagation characteristics of electromagnetic fields mainly include effective medium theory (EMT), finitedifference time-domain method (FDTD), finite element method (FEM) and rigorous coupled wave method (RCWA). Because of the period of the antireflection microstructure is much smaller than the incident wavelength, effective medium theory (EMT) were chose to do the analysis. This theory explains that when an electromagnetic wave propagated through a grating which is much smaller than it, there would be only one reflection and one transmission wave in the zero order grating. The following grat- 
ing equation determines whether a diffraction order could propagates or not,

$$
n_{s} \sin \theta_{m}=\frac{m \lambda}{\Lambda}+n_{i} \sin \theta_{i}
$$

$\mathrm{n}_{\mathrm{i}}$ represents the refraction index when the light incidentting to the medium, $\mathrm{n}$ indicates the refraction index when the light transmitting through the medium (when it come to the reflection diffraction orders, $n=n_{i}$. As for the transmission diffraction, $\mathrm{n}$ would be the same as the substrate's refraction index.) Using $\theta_{\mathrm{m}}$ and $\theta_{\mathrm{i}}$ to represent the angle of the $\mathrm{m}$ order and the angle of incident wave which are measured from the grating's surface normal, respectively. $\lambda$ is on behalf of the wavelength of the incident free space, and $\Lambda$ is the grating period. When the high order diffraction fields are forced to be evanescent, a zero order grating is produced. The inequality of the periodic of $2 \mathrm{D}$ grating and wavelength would be determined by

$$
\begin{aligned}
\frac{\Lambda_{x}}{\lambda} & <\frac{1}{\left[\max \left(n_{s}^{2}, n_{i}^{2}\right)-\left(n_{i} \sin \theta_{i} \sin \phi_{i}\right)^{2}\right]^{1 / 2}+\left|n_{i} \sin \theta_{i} \cos \phi_{i}\right|} \\
\frac{\Lambda_{\mathrm{y}}}{\lambda} & <\frac{1}{\left[\max \left(n_{s}^{2}, n_{i}^{2}\right)-\left(n_{i} \sin \theta_{i} \sin \phi_{i}\right)^{2}\right]^{1 / 2}+\left|n_{i} \sin \theta_{i} \cos \phi_{i}\right|}
\end{aligned}
$$

Max were used to refer the maximum value of either $n_{\mathrm{s}}$ or $\mathrm{n}_{\mathrm{i}}$. The period of the grating divided by the wavelength of operation $(\Lambda \lambda)$ can be used to define the normalized grating period. When the grating is designed at all incidence for the experiment, the normalized period of the grating reduces to,

$$
\Lambda<\frac{\lambda}{\max \left(n_{s}, n_{i}\right)+n_{i}}
$$

The refractive index of $\mathrm{ZnSe}$ from 2 to $15 \mu \mathrm{m}$ is about 2.4 , so 2.4 was brought into inequality above. We choose 9 $\mu \mathrm{m}$ as the incident wavelength, then we got the period of the grating, $\Lambda=2.9 \mu \mathrm{m}$. In order to calculated the value of the minimum depth of the structured, following formula were used,

$$
d_{\min }=\frac{\lambda}{4 \sqrt{n_{s} n_{i}}}
$$

In this way, the minimum depth of antireflection microstructure is about $1.45 \mu \mathrm{m}$. Processing capability was
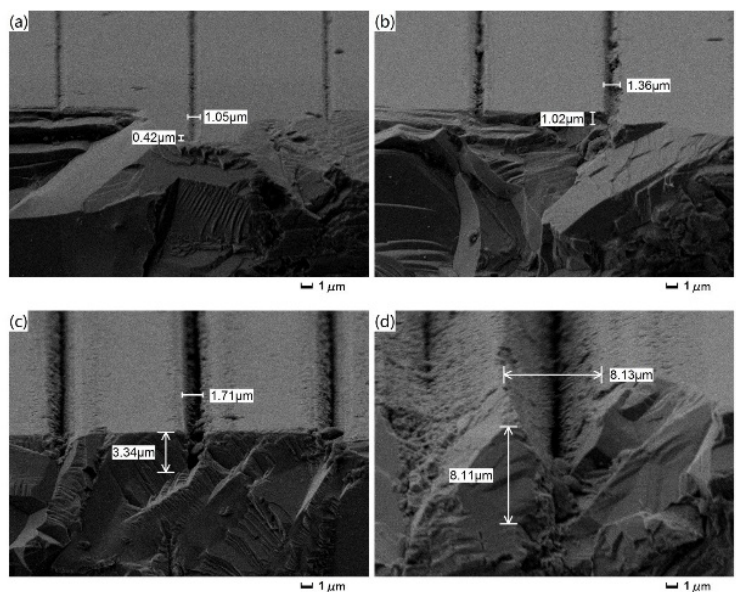

Fig.1 Dependence of width and depth of the line structures on laser power. (a) $0.13 \mathrm{~mJ} / \mathrm{cm}^{2}$; (b) $0.2 \mathrm{~mJ} / \mathrm{cm}^{2}$; (c) $0.48 \mathrm{~mJ} / \mathrm{cm}^{2}$ and (d) $0.87 \mathrm{~mJ} / \mathrm{cm}^{2}$. proved in the structure we prepared in Figure 1. The fluence of the laser were $0.13 \mathrm{~mJ} / \mathrm{cm}^{2}, 0.2 \mathrm{~mJ} / \mathrm{cm}^{2}, 0.48$ $\mathrm{mJ} / \mathrm{cm}^{2}, 0.87 \mathrm{~mJ} / \mathrm{cm}^{2}$ respectively. It could be seen that the width of the structures were getting wider as the laser power increasing. The depth of the structures were getting deeper as the laser power increasing. The experiment of processing effects at different fluence facilitates the later fabricating of antireflection microstructures. Therefore, required antireflection microstructure can be prepared easily by adjusting the laser power.

Figure 2 shows the optical path of the experiment. Antireflection microstructured surfaces were fabricated by a commercial Ti:Sapphire laser (Spectra-Physics), with the ability to deliver $120 \mathrm{fs}$ pulses at $2.5 \mathrm{kHz}$ repetition rate at a $800 \mathrm{~nm}$ center wavelength. The ablation fluence of the laser was $0.174 \mathrm{~mJ} / \mathrm{cm}^{2}$ during the experiment. Due to the diffraction limit of the interaction between the laser and the material, BBO crystal were used to double the frequency. With an $80 \times$ objective lens, collimated beam was focused on a piece of $\mathrm{ZnSe}$. To reach the repeatability and accuracy of each experiment, a variable neutral-density filter were inserted between the laser and the lens. We used a nanometer precision piezo stage (PI P-622 ZCD) and a galvano mirror to control the movement of the material and the laser beam. 3D geometry of various microstructure were firstly designed by the C programming language, and used it for our manufacturing. The sample is attached to the piezo stage by water. And the top surface of it can be seen clearly by a silicon charge-coupled device (CCD) (HDGY500), with a spectral response range from 400nm to $1000 \mathrm{~nm}$. After trying different fabricating parameters, we fixed single pulse exposure time at $1000 \mu$ s and the scanning interval is $100 \mathrm{~nm}$. Acetone, alcohol and deionized water were used before and after the fabrication to remove the dust and the debris.

\section{Results}

Four different arrangements of antireflection microstructure were obtained by a scanning electron microscope (SEM) which were showed in Figure 3. Pyramid-square, pyramid-hexagon, cone-square and cone-hexagon arrangements of antireflection microstructure were fabricated with

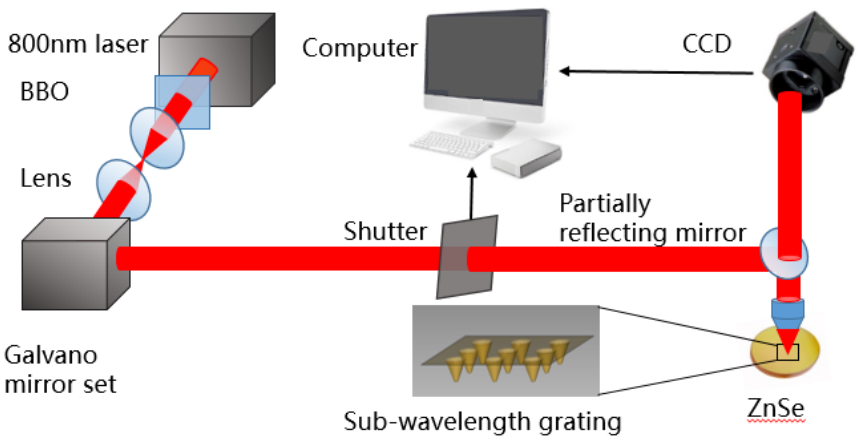

Fig.2 This is an illustration of the femtosecond laser fabricating anti-reflection microstructured surface on the $\mathrm{ZnSe}$. 

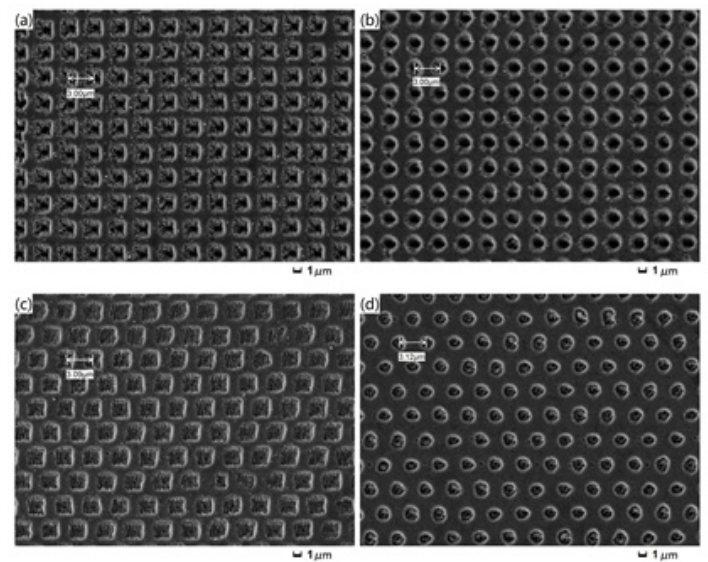

Fig.3 The SEM image of the sub-wavelength antireflection microstructure. (a) Pyramid-square; (b) Pyramid-hexagon; (c) Cone-square and (d) Cone-hexagon.

different designs. The electrical conductivity of the sample were improved by an $\mathrm{Au}$ film. This $10 \mathrm{~nm}$ film was sputtered with an auto fine coater (JFC-1600; JEOL) at a current of $20 \mathrm{~mA}$ for $60 \mathrm{~s}$ before the SEM. The subwavelength microstructures had a period of $3 \mu \mathrm{m}$ and a depth of $2 \mu \mathrm{m}$ which were of good surface morphology. Pyramid and square structure can cause a slightly different in transmission spectrum. Fabricating time can be reduced by a wide margin with the inverted structure we design. Which is 5 minutes during the experiment. In order to improve the femtosecond laser direct writing efficiency, field lens with the focal length of $10 \mathrm{~cm}$ can also be used during the fabrication. With this kind of fabricating equipment, $1 \mathrm{~cm} \times 1 \mathrm{~cm}$ antireflection microstructure can be produced within 20 minutes at the same situation.

Highly magnified laser scanning confocal microscopy (LSCM) were also used to reveal the three dimensional profile of the antireflection microstructure. The depth and the width of pyramid-square, pyramid-hexagon, conesquare and cone-hexagon arrangements of antireflection microstructure were shown in Figure 4. The horizontal and vertical profile information of the antireflection
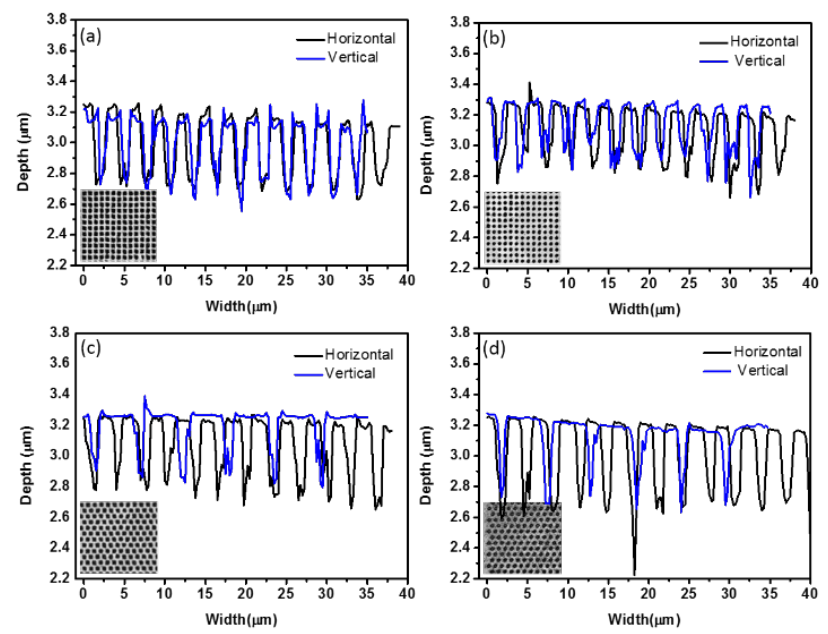

Fig.4 The LSCM image and the profile information of the sub-wavelength antireflection microstructure. (a) Pyramidsquare; (b) Pyramid-hexagon; (c) Cone-square and (d) Conehexagon.

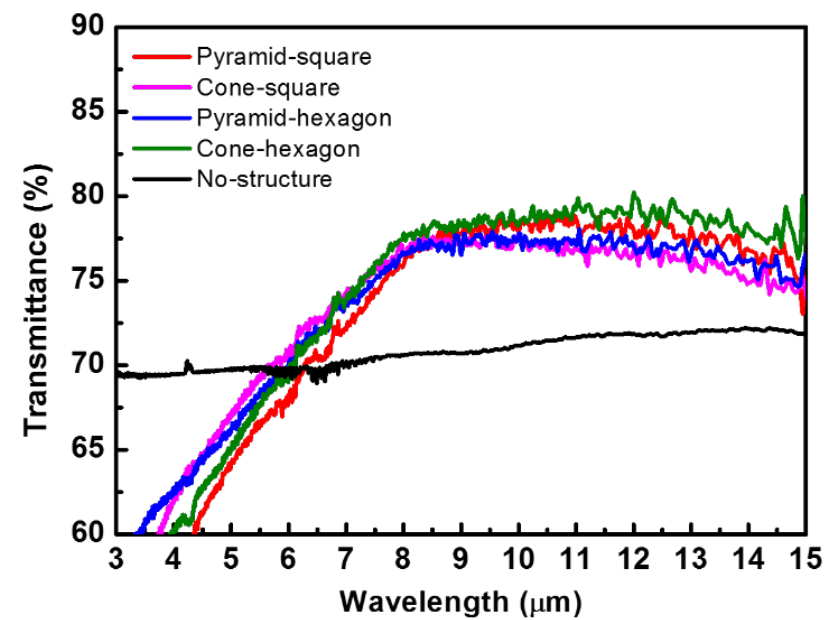

Fig.5 Measured transmittance of fabricated sub-wavelength antireflection microstructure on the $\mathrm{ZnSe}$ using Fourier transform infrared spectrometer.

microstructure were given in black and blue line.It can be seen from the figure that the sturcture is uniform and consistent with the design.

The transmittance of the pure $\mathrm{ZnSe}$ and the structures were carried out with a Fourier transform infrared spectrometer (FT-IR, Nicolet 6700), which were based on the principle of Fourier transform. With the help of the Origin 9.0, the transmittance of different structures were shown in Figure 5. The transmittance of the pure $\mathrm{ZnSe}$ is only about $70 \%$. With antireflection microstructure, the transmittance were improved from $6 \mu \mathrm{m}$ to $15 \mu \mathrm{m}$. Solid lines of different colors represents different arrangements of antireflection microstructure. The red, pink, blue, green line represent pyramid-square, cone-square, pyramid-hexagon and conehexagon arrangement of the antireflection microstructure respectively. It has been shown from the green line that the cone-hexagon structure has a slightly higher transmittance, which were about $80 \%$. Figure 6 is the simulation of the inverted cone-square structure calculated by Virtual Lab. In the simulation, the wavelength range of the incident light were set from $12 \mu \mathrm{m}$ to $15 \mu \mathrm{m}$.

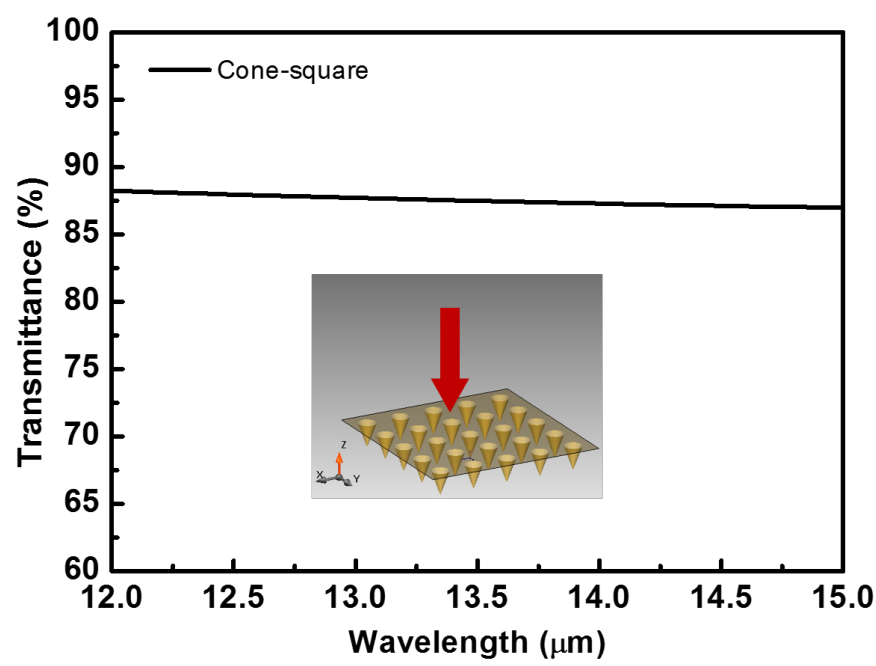

Fig.6 Simulation result of the inverted cone-square transmittance performance. 
The transmittance of the cone-square were about $87 \%$. It could be seen that the experiment result were in good accord with the simulation.

\section{Conclusions}

In conclusion, micron scale antireflection microstructured surface can be manufactured by femtosecond laser direct writing technology on hard material. With this kind of processing method, the transmittance of the sample can be improved economically and quickly. Different arrangements of antireflection microstructure have slightly different antireflection effect. The transmittance of $\mathrm{ZnSe}$ were improved from $70 \%$ to about $80 \%$ in the Mid-infrared region. In order to further improve the transmittance of the sample, both side structure can be prepared. This kind of structures may have a good application prospects in Midinfrared imaging and measurement.

\section{Acknowledgments}

This work was supported by National Key R\&D Program of China (2017YFB1104600) and National Nature Science Foundation of China (NSFC) (61590930, 21473076, 61605055 and 61435005).

\section{References}

[1] J. Kvietkova, B. Daniel and M. Hetterich: Thin Solid Films, 455, (2004) 228.

[2] M. A. Abdel-Rahim, M. M. Hafiz and A. E. B. Alwany: Opt. Laser Technol., 47, (2013) 88.

[3] N. Zeiri, A. B. Nasrallah, N. Sfina and M. Said: Infrared Phys. Techn., 64, (2014) 33.

[4] Y. Qu, Z. H. Kang, T. J. Wang, Y. G. Jiang, Yu. M.Andreev and J. Y. Gao: Laser Phys. Lett., 4, (2010) 238.

[5] P. Kannappan, K. Baskar, J. B. M. Krishna, K. Asokan, C. L. Dong and C. L. Chen: Mater. Sci. Semicon. Proc., 36, (2015) 140.

[6] D. C. Harris: Infrared Phys. Technol., 39, (1998) 185.

[7] H. H. Li: Journal of Physical and Chemical Reference Data, 13, (1984) 103.

[8] M. Shokooh-Saremi and M. M. Mirsalehi: Appl. Opt., 44, (2005) 3877.

[9] A. L. Pénard, T. Gacoin and J. P. Boilot: Acc. Chem. Res., 38, (2007) 895.

[10] L. Martinu and D. Poitras: J. Vac. Sci. Technol. A, 18, (2000) 2619.

[11]H. K. Raut, V. A. Ganesh and A. S. Nair: Energy Environ. Sci., 4, (2011) 3779

[12] S. P. Min, Lee. A. Youngmin and K. K Jim: Chem. Mater.,17, (2005)

[13] J. Amirloo, S. S. Saini and M. Dagenais, J. Vac. Sci. Technol. A, 34, (2016) 061505.

[14] Y. Ono, Y. Kimura, Y. Ohta and N. Nishida: Appl. Opt., 26, (1987) 1142.

[15] W. H. Miller, G. D. Bernard, J. L. Allen: Science, 162, (1968) 760.

[16] A. Gombert, K. Rose, A. Heinzel A, W. Horbelt, C. Manecke, B. Bläsi and V. Wittwer: Sol. Energy Mater. Sol. Cells, 54, (1998) 333.

[17] T. Lohmüller, M. Helgert, M. Sundermann, R. Brunner and J. P. Spatz: Nano Lett., 8, (2008) 1429.
[18] G. Q. Du, Q. Yang, F. Chen, H. W. Liu, Z. F. Deng, H. Bian, S. G. He, J. H. Si, X. W. Meng and X. Hou: Opt. Lett., 37. (2012) 4404.

[19] L. Wang, Q. D. Chen, X. W. Cao, R. Buividas, X. Wang, X. W. Wang, S. Juodkazis and H. B. Sun: Light Sci. Appl., 6, (2017), e17112.

[20] R. Kammel, R. Ackermann, J. Thomas, J, Götte, S. Skupin, A. Tünnermann and S. Nolte: Light Sci. Appl., 3, (2014) e169.

[21] H. Imamoto, S. Kanehira, X. Wang, K. Kametani, M. Sakakura, Y. Shimotsuma, K. Miura, and K. Hirao: Opt. Lett., 36, (2011) 1176

[22] S. Juodkazis, K. Nishimura, H. Misawa, T. Ebisui, R. Waki, S. Matsuo and T. Okada: Adv. Mater., 18, (2006) 1361.

[23] K. Sugioka, Y. Cheng: Light Sci. Appl., 3, (2014) e149.

[24]X. Q. Liu, Q. D. Chen, K. M. Guan, Z. C. Ma, Y. H. $\mathrm{Yu}, \mathrm{Q}$. K. Li, Z. N. Tian and H. B. Sun: Laser Photon. Rev., 11, (2017) 1600115.

[25] H. Wang, Y. L. Zhang, W. Wang, H. Ding and H. B Sun: Laser Photon. Rev., 11, (2017) 1600116.

[26] L. Jiang, A. D Wang, B. Li, T. H. Cui and Y. F. Lu: Light Sci. Appl., 7, (2018) 17134.

(Received: June 27, 2018, Accepted: April 2, 2019) 